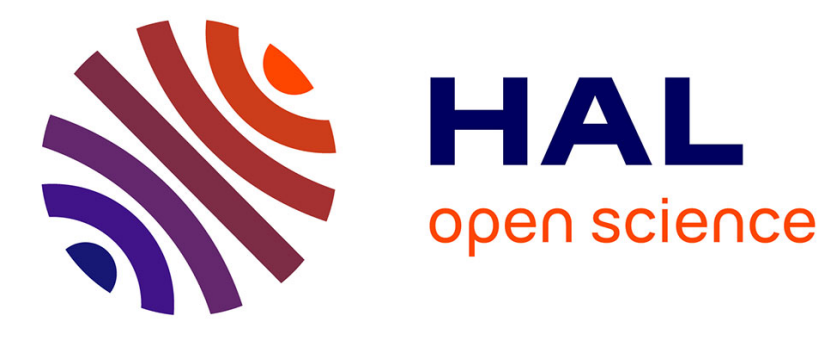

\title{
Modelling of a vibro-impact capsule system
}

Yang Liu, Marian Wiercigroch, Ekaterina Pavlovskaia, Hongnian Yu

\section{To cite this version:}

Yang Liu, Marian Wiercigroch, Ekaterina Pavlovskaia, Hongnian Yu. Modelling of a vibroimpact capsule system. International Journal of Mechanical Sciences, 2013, 66, pp.2-11. 10.1016/j.ijmecsci.2012.09.012 . hal-01510831

\section{HAL Id: hal-01510831 \\ https://hal.science/hal-01510831}

Submitted on 20 Apr 2017

HAL is a multi-disciplinary open access archive for the deposit and dissemination of scientific research documents, whether they are published or not. The documents may come from teaching and research institutions in France or abroad, or from public or private research centers.
L'archive ouverte pluridisciplinaire HAL, est destinée au dépôt et à la diffusion de documents scientifiques de niveau recherche, publiés ou non, émanant des établissements d'enseignement et de recherche français ou étrangers, des laboratoires publics ou privés. 


\title{
Modelling of a vibro-impact capsule system
}

\author{
Yang Liu ${ }^{a}$, Marian Wiercigroch ${ }^{a}$, Ekaterina Pavlovskaia ${ }^{a}$, Hongnian Yu ${ }^{b}$ \\ Centre for Applied Dynamics Research, School of Engineering, King's College, University of Aberdeen, UK \\ ${ }^{\mathrm{b}}$ School of Design, Engineering and Computing, Bournemouth University, Bournemouth, UK
}

\begin{abstract}
The model of a vibro-impact capsule system is studied in this paper to provide a fundamental understanding of its dynamics for potential medical and engineering applications. The system consists of a capsule having a main body which interacts with an internal mass driven by a harmonic excitation. Impacts occur when the internal mass contacts a weightless plate connected to the capsule main body. The main function of the system is to drive the capsule by overcoming resistance forces from the environment. Our bifurcation studies show that the dynamic behaviour of the system is mainly periodic, and the best progression can be determined through this analysis by a careful choice of the system parameters. Energy consumption is also considered and it is found that the parameters for the best progression and for the minimum energy consumption are different, and therefore, a trade-off between the best progression and the energy consumption is required to optimize the system behaviour.
\end{abstract}

\author{
Keywords: \\ Vibro-impact \\ Stick-slip \\ Dry friction \\ Capsule dynamics
}

\section{Introduction}

In recent years, mobile mechanisms which are driven by autogenous internal force have attracted significant attention from researchers in different disciplines fueled by their broad applications in medical inspection, engineering diagnosis, and disaster rescues. The basic idea originally proposed by Chernousko [1] is that the rectilinear motion of a system can be engineered using a periodically driven internal mass interacting with the main body in the presence of dry friction (see Fig. 1). The advantage of this method is that no external driving mechanism is required, so the system can be encapsulated and move independently in the complex environment. Imagine for example, a miniaturized medical capsule which is moving inside human body by adopting this method. In this case, many complications induced by external driving mechanism, e.g. [2,3], can be avoided. However, understanding of the dynamics and efficient control of such driving mechanism are critical, and they have to be carefully analysed and designed for the system to be able to complete its tasks.

This paper studies mathematical model of a new capsule system, which employs an internal vibro-impact force for driving. Similar mobile mechanisms driven by self-propulsion and dry friction have been previously considered in robotics. For instance, Vartholomeos and Papadopoulos [5] designed a micro-robotic platform which was able to perform translational and rotational motions by employing vibration micro-actuators. In [6], horizontal and vertical oscillations of internal masses within carrying body were utilised to create a vibration-driven mechanism. It has been shown that the motion of the system can be controlled by the phase shift between masses oscillations in horizontal and vertical directions when the frequency of these oscillations is the same. An example of an unbalanced rotor acting as a vibration exciter was considered and optimal frequency of the oscillation was determined to maximise the average velocity. Li et al. [7] theoretically studied an optimal motion for an internal mass moving inside a capsule, and Su et al. [8] designed a capsule system driven by an electromagnetic force and investigated it experimentally. Furthermore, Liu et al. [9] proposed a mobile cart which may exhibit planar motions on a surface when excited by two parallel pendulums rotating in a specified manner. Fang and $\mathrm{Xu}[10,11]$ studied dynamics of a mobile system in a resistive medium by controlling an internal mass. The common issue for the capsule-type mechanisms (e.g. $[6-8,10,11]$ ) is that, the capsule progression is constrained by the motion of internal mass, and driving such a mass within a limited dimension is extremely difficult in experiment. On the other hand, the problem for the large-scale mobile platforms (e.g. $[5,9]$ ) is that they are difficult to be miniaturized and therefore have limited applicability. To address these issues, we propose a new mechanism, where in addition to the harmonic oscillations of the internal mass, the soft impacts between this mass and the capsule are used in order to enhance the progression of the entire system. 


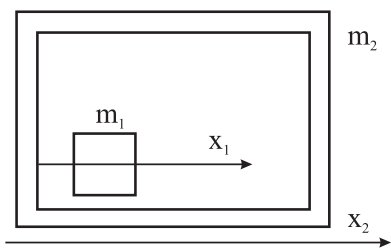

b

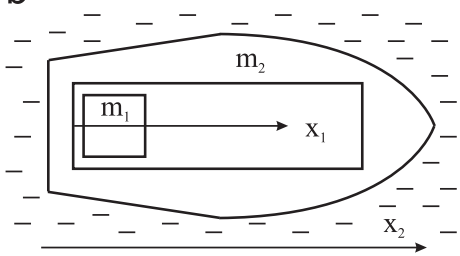

Fig. 1. Physical models of the capsule system: (a) on a resistive surface and (b) in a resistive medium (adopted from [4]).

This paper proposes a vibro-impact capsule system which is inspired by a drifting oscillator studied by Pavlovskaia et al. [12]. As shown in [12], the behaviour of this impact oscillator may vary from periodic to chaotic due to nonsmooth nature of the underlying equations of motion and the progressive motion of the system is possible in one direction. For our proposed system, the case is more complicated, since the rectilinear motion of the system is bidirectional. So bifurcation studies are carried out in the paper in order to provide a better insight for design of such system. The purpose of this study is to analyse a physical model of our proposed capsule system which can be used, for example, for capsule endoscopy [13], where a swallowable capsule equipped with a miniature camera is able to move inside human body and help to determine gastrointestinal symptoms. The applications of such system can be even broader extending to pipeline fault diagnosis, life searching in collapsed building, and others.

This paper is organized as follows. In Section 2, mathematical modelling of the vibro-impact capsule system is described. In Section 3, nonlinear dynamic analysis is presented, and the periodic and chaotic regimes of the system under varying control parameters are discussed. In particular, the best progressions under different control parameters are obtained. In Section 4, energy consumption of the system is considered and discussed. Finally, some concluding remarks are drawn in Section 5.

\section{Mathematical modelling}

We consider a two degrees-of-freedom system, which is composed of a rigid capsule containing within an attached internal mass as shown in Fig. 2. The movable internal mass $m_{1}$ is driven by an external harmonic force with amplitude $P_{d}$ and frequency $\Omega$ generated by a linear actuator (e.g. piezoelectric actuator). The actuator contains a movable part connected to the internal mass and a fixed part mounted on the rigid capsule $m_{2}$. We simplify the model of the actuator here and represent the interaction between the mass and the capsule by using a linear spring with stiffness $k_{1}$ and a viscous damper with damping coefficient $c$. A weightless plate is connected to the capsule by a secondary linear spring with stiffness $k_{2} . X_{1}$ and $X_{2}$ represent the absolute displacements of the internal mass and the capsule, respectively. The internal mass will contact the plate when the relative displacement $X_{1}-X_{2}$ is larger or equals the gap $G$. The bidirectional motion of the capsule occurs when the force acting on the capsule from the internal mass exceeds the threshold of the dry friction force $P_{f}$ between the capsule and the environment surface.

\subsection{Equations of motion}

As the system may operate in bidirectional stick-slip phases, the relative displacement $X_{1}-X_{2}$ and the velocity of the capsule $\dot{X}_{2}$ have to be closely monitored to ensure the appropriate switches between various sets of the equations of motion which

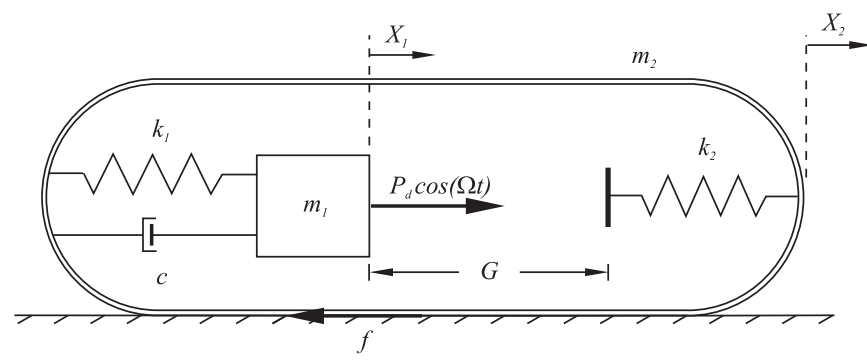

Fig. 2. Physical model of the vibro-impact capsule system.

are discussed below. In this study, Coulomb friction model is used to calculate the frictional force between the capsule and the sliding surface

$f= \begin{cases}0, & \dot{X}_{2}=0, \\ -\operatorname{sign}\left(\dot{X}_{2}\right) \cdot P_{f}, & \dot{X}_{2} \neq 0,\end{cases}$

where $P_{f}=\mu\left(m_{1}+m_{2}\right) g, \mu$ is the friction coefficient between the capsule and the environment surface, and $g$ is the acceleration due to gravity.

For the case when the internal mass and the plate are not in contact $\left(X_{1}-X_{2}<G\right)$, the motion of the internal mass is governed by

$m_{1} \ddot{X}_{1}=P_{d} \cos (\Omega t)+k_{1}\left(X_{2}-X_{1}\right)+c\left(\dot{X}_{2}-\dot{X}_{1}\right)$

The capsule is either stationary

$\dot{X}_{2}=0$

when the force acting on the capsule from the internal mass is smaller than the threshold of the dry friction force $\left|k_{1}\left(X_{2}-X_{1}\right)+c\left(\dot{X}_{2}-\dot{X}_{1}\right)\right|<P_{f}$, or moves $\left(\dot{X}_{2} \neq 0\right)$ as described by the following equation:

$m_{2} \ddot{X}_{2}=-\operatorname{sign}\left(\dot{X}_{2}\right) \cdot P_{f}-k_{1}\left(X_{2}-X_{1}\right)-c\left(\dot{X}_{2}-\dot{X}_{1}\right)$.

It is important to note that once the force acting on the capsule reaches the critical level, i.e. $\left|k_{1}\left(X_{2}-X_{1}\right)+c\left(\dot{X}_{2}-\dot{X}_{1}\right)\right|=P_{f}$, the capsule begins to move and the direction of the dry friction force acting on the capsule at this moment is opposite to the elastic force acting on the capsule. In this moment the dry friction force is calculated as

$f=-\operatorname{sign}\left(k_{1}\left(X_{2}-X_{1}\right)+c\left(\dot{X}_{2}-\dot{X}_{1}\right)\right) \cdot P_{f}$.

When the internal mass and the plate are in contact $\left(X_{1}-X_{2} \geq G\right)$, the motion of the internal mass is governed by

$m_{1} \ddot{X}_{1}=P_{d} \cos (\Omega t)+k_{1}\left(X_{2}-X_{1}\right)+c\left(\dot{X}_{2}-\dot{X}_{1}\right)-k_{2}\left(X_{1}-X_{2}-G\right)$.

The capsule is either stationary as Eq. (3) (in this case $\left.\left|k_{1}\left(X_{2}-X_{1}\right)+c\left(\dot{X}_{2}-\dot{X}_{1}\right)-k_{2}\left(X_{1}-X_{2}-G\right)\right|<P_{f}\right)$ or moving $\left(\dot{X}_{2} \neq 0\right)$ as described by the following equation:

$m_{2} \ddot{X}_{2}=k_{2}\left(X_{1}-X_{2}-G\right)-\operatorname{sign}\left(\dot{X}_{2}\right) \cdot P_{f}-k_{1}\left(X_{2}-X_{1}\right)-c\left(\dot{X}_{2}-\dot{X}_{1}\right)$.

Again, once the force acting on the capsule reaches the critical level, i.e. $\left|k_{1}\left(X_{2}-X_{1}\right)+c\left(\dot{X}_{2}-\dot{X}_{1}\right)-k_{2}\left(X_{1}-X_{2}-G\right)\right|=P_{f}$, the capsule begins to move and the direction of the dry friction force acting on the capsule at this moment is opposite to the elastic force acting on the capsule. In this moment the dry friction force is calculated as

$f=-\operatorname{sign}\left(k_{1}\left(X_{2}-X_{1}\right)+c\left(\dot{X}_{2}-\dot{X}_{1}\right)-k_{2}\left(X_{1}-X_{2}-G\right)\right) \cdot P_{f}$.

It is clear that the motion of the capsule system can be very complex and in general may consists of four phases (i.a) stationary capsule when the internal mass and the plate are not in 
contact, (i.b) moving capsule without contact, (ii.a) stationary capsule with contact, and (ii.b) moving capsule with contact.

\subsection{Non-dimensional equations}

We introduce the following non-dimensional variables and parameters:

$\tau=\Omega_{0} t, \quad x_{i}=\frac{k_{1}}{P_{f}} X_{i}, \quad y_{i}=\frac{d x_{i}}{d \tau}=\frac{k_{1}}{\Omega_{0} P_{f}} \dot{X}_{i}$,

$\dot{y}_{i}=\frac{d y_{i}}{d \tau}=\frac{k_{1}}{\Omega_{0}^{2} P_{f}} \ddot{X}_{i}, \quad \Omega_{0}=\sqrt{\frac{k_{1}}{m_{1}}}, \quad \omega=\frac{\Omega}{\Omega_{0}}, \quad \alpha=\frac{P_{d}}{P_{f}}$,

$\xi=\frac{c}{2 m_{1} \Omega_{0}}, \quad \delta=\frac{k_{1}}{P_{f}} G, \quad \beta=\frac{k_{2}}{k_{1}}, \quad \gamma=\frac{m_{2}}{m_{1}}$,

where $i=1,2$. Then the equations of motion is re-written as follows.

\subsection{1. (i.a) Stationary capsule without contact}

If the relative displacement between the mass and the capsule is smaller than the gap,

$x_{1}-x_{2}<\delta$

the mass has no contact with the plate. The motion of the mass is then described by

$\dot{x}_{1}=y_{1}$,

$\dot{y}_{1}=\alpha \cos (\omega \tau)+\left(x_{2}-x_{1}\right)+2 \xi\left(y_{2}-y_{1}\right)$.

If the force on the capsule from the spring and damper is smaller or equals to the threshold of the dry friction force,

$\left|\left(x_{2}-x_{1}\right)+2 \xi\left(y_{2}-y_{1}\right)\right| \leq 1$

the capsule is stationary,

$\dot{x}_{2}=0, \quad \dot{y}_{2}=0$.

Once the force acting on the capsule reaches the threshold $\left|\left(x_{2}-x_{1}\right)+2 \xi\left(y_{2}-y_{1}\right)\right|=1$, the capsule begins to move, and the direction of the dry friction force is determined by the elastic force acting on the capsule, so it is equal to $-\operatorname{sign}\left(\left(x_{2}-x_{1}\right)+\right.$ $\left.2 \xi\left(y_{2}-y_{1}\right)\right)$.

\subsection{2. (i.b) Moving capsule without contact}

If the force on the capsule from the spring and damper is larger than its dry friction force,

$\left|\left(x_{2}-x_{1}\right)+2 \xi\left(y_{2}-y_{1}\right)\right|>1$

the capsule is moving $\left(y_{2} \neq 0\right)$ as

$\dot{x}_{2}=y_{2}$,

$\dot{y}_{2}=\left(-\operatorname{sign}\left(y_{2}\right)-\left(x_{2}-x_{1}\right)-2 \xi\left(y_{2}-y_{1}\right)\right) / \gamma$

and the motion of the mass is described by Eq. (10).

\subsection{3. (ii.a) Stationary capsule with contact}

The impact occurs when the relative displacement between the mass and the capsule is larger or equals to the gap,

$x_{1}-x_{2} \geq \delta$

In this case, the motion of the mass is governed by the following differential equations:

$\dot{x}_{1}=y_{1}$,

$\dot{y}_{1}=\alpha \cos (\omega \tau)+\left(x_{2}-x_{1}\right)+2 \xi\left(y_{2}-y_{1}\right)-\beta\left(x_{1}-x_{2}-\delta\right)$.

The capsule remains stationary as described by Eq. (12), when the force acting on the capsule from the springs and damper is smaller or equals to its dry friction

$\left|\left(x_{2}-x_{1}\right)+2 \xi\left(y_{2}-y_{1}\right)-\beta\left(x_{1}-x_{2}-\delta\right)\right| \leq 1$.

Once the force acting on the capsule reaches the threshold $\left|\left(x_{2}-x_{1}\right)+2 \xi\left(y_{2}-y_{1}\right)-\beta\left(x_{1}-x_{2}-\delta\right)\right|=1$, the capsule begins to move, and the direction of the dry friction force is determined by the force acting on the capsule from the springs and damper, so it is equal to $-\operatorname{sign}\left(\left(x_{2}-x_{1}\right)+2 \xi\left(y_{2}-y_{1}\right)-\beta\left(x_{1}-x_{2}-\delta\right)\right)$.

2.2.4. (ii.b) Moving capsule with contact

If the force on the capsule is larger than its dry friction force,

$\left|\left(x_{2}-x_{1}\right)+2 \xi\left(y_{2}-y_{1}\right)-\beta\left(x_{1}-x_{2}-\delta\right)\right|>1$

the capsule is moving $\left(y_{2} \neq 0\right)$ as described by the following equations:

$\dot{x}_{2}=y_{2}$,

$\dot{y}_{2}=\left[\beta\left(x_{1}-x_{2}-\delta\right)-\operatorname{sign}\left(y_{2}\right)-\left(x_{2}-x_{1}\right)-2 \xi\left(y_{2}-y_{1}\right)\right] / \gamma$

and the motion of the mass is described by Eq. (16).

Let us define a set of auxiliary functions,

$H_{1}=H\left(\left|\left(x_{2}-x_{1}\right)+2 \xi\left(y_{2}-y_{1}\right)\right|-1\right)$,

$H_{2}=H\left(\left|\left(x_{2}-x_{1}\right)+2 \xi\left(y_{2}-y_{1}\right)-\beta\left(x_{1}-x_{2}-\delta\right)\right|-1\right)$,

$H_{3}=H\left(x_{1}-x_{2}-\delta\right)$,

where $H(\cdot)$ is the Heaviside function.

Finally, the comprehensive equations of motion for the vibroimpact capsule system can be written as

$\dot{x}_{1}=y_{1}$,

$\dot{y}_{1}=\alpha \cos (\omega \tau)+\left(x_{2}-x_{1}\right)+2 \xi\left(y_{2}-y_{1}\right)-H_{3} \beta\left(x_{1}-x_{2}-\delta\right)$,

$\dot{x}_{2}=y_{2}\left(H_{1}\left(1-H_{3}\right)+H_{2} H_{3}\right)$,

$\dot{y}_{2}=\left(H_{1}\left(1-H_{3}\right)+H_{2} H_{3}\right)\left(-\operatorname{sign}\left(y_{2}\right)-\left(x_{2}-x_{1}\right)-2 \xi\left(y_{2}-y_{1}\right)\right.$

$\left.+H_{3} \beta\left(x_{1}-x_{2}-\delta\right)\right) / \gamma$.

\section{Nonlinear dynamic analysis}

The purpose of the considered system is to drive the capsule in a desired direction overcoming the resistance force from the environmental surface. Although there are some similarities in principals of operation between this system and the drifting oscillator studied in $[12,14]$, the main difference is that the motion of the drifting oscillator is unilateral (i.e. in no circumstances the slider can move backwards), while our system is capable of bidirectional motion in horizontal direction. The numerical calculations were carried out using the first-order Euler method with a fixed time step calculated as $1 / 10^{4}$ of the period of the external excitation.

\subsection{Period-1 motion}

A typical time history of the system displacement is presented in Fig. 3, where the displacement of the mass (solid black line) and the capsule (dash red line) are shown. To show the details, a zoom up of the time history between 52.4 and 57.9 (Interval A) is depicted in Fig. 4, where a sequence of four phases for a periodone motion is shown during one period $T$ of the external loading. This typical pattern comprised the following phases:

- Phase I-the mass and the plate are in contact, and the capsule is stationary. 


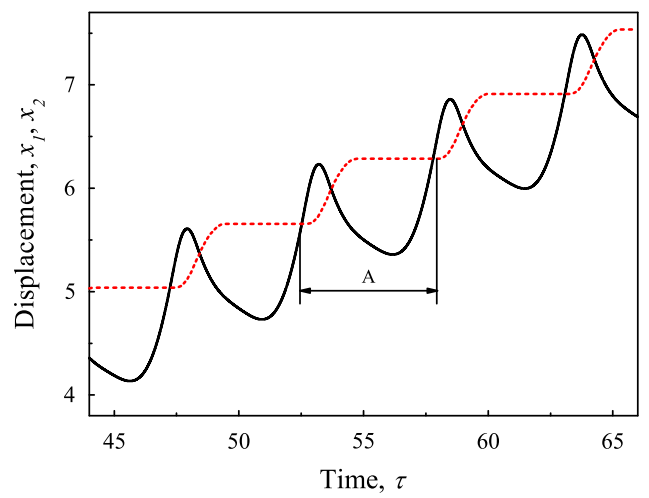

Fig. 3. (colour online) Time histories of displacements of the mass, $x_{1}$ (solid black line), and the capsule, $x_{2}$ (dash red line), calculated for $\alpha=0.8, \omega=1.19, \delta=0.02$, $\xi=0.05, \beta=6.6$, and $\gamma=3.0$.

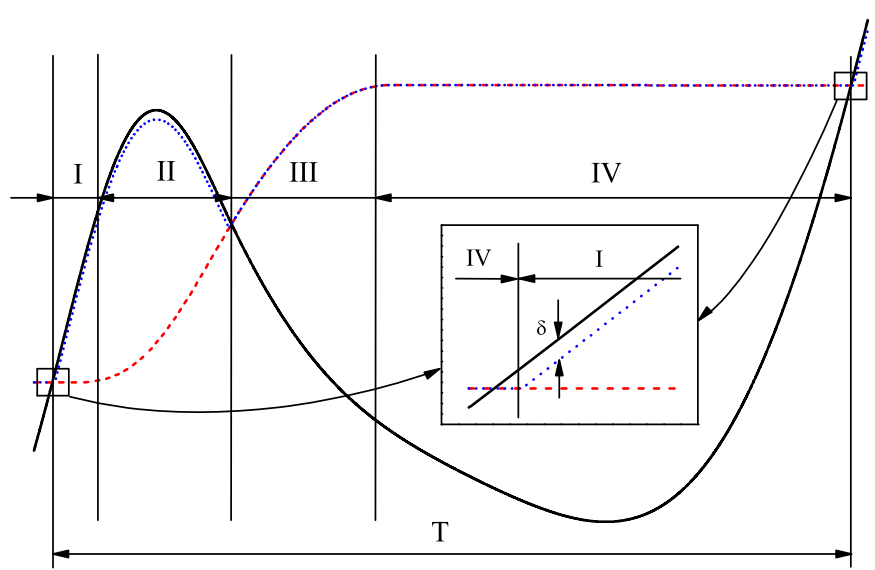

Fig. 4. (colour online) Time histories of displacements of the mass, $x_{1}$ (solid black line), the capsule, $x_{2}$ (dash red line), and the plate (dot blue line) in four phases for a period-one motion, calculated for $\alpha=0.8, \omega=1.19, \delta=0.02, \xi=0.05, \beta=6.6$, and $\gamma=3.0$.

- Phase II-the mass and the plate are in contact, and the capsule is moving.

- Phase III-the mass and the plate are not in contact, and the capsule is moving.

- Phase IV-the mass and the plate are not in contact, and the capsule is stationary.

As seen from Fig. 4, the period-one motion starts when the mass and the plate are just in contact while the capsule is stationary. A blow-up window clearly shows the displacements of the mass, the capsule, and the plate at the beginning of Phase I. Once the force acting on the capsule from the springs and damper reaches the threshold of dry friction, the capsule starts to move and Phase II begins. The mass and the plate separates in the beginning of Phase III, and then the plate moves forward together with the capsule. When the force acting on the capsule becomes smaller than the threshold of dry friction, the Phase IV begins and the capsule and the plate remain stationary until the end of this phase.

\subsection{Bifurcation analysis}

In order to gain an understanding of the system dynamics, the bifurcation analysis is carried out next. First we consider the behaviour of the system for varying mass ratio, $\gamma$. The bifurcation diagram presented in Fig. 5(a) shows the velocity $y_{1}^{*}$, which is a projection of the Poincare map on the $y_{1}$ axis. In contrast to the displacements of the mass and the capsule, velocities are bounded and therefore $y_{1}^{*}$ has been chosen to construct the bifurcation diagram. The calculations were run for 300 cycles of the external loading and, to ensure the steady state response, the data for the first 100 cycles were omitted, whereas the next 200 values of the velocity, $y_{1}^{*}$ were plotted.

As can be seen in Fig. 5(a), the system has period-one motion for all the values of mass ratio, $\gamma$. Additional windows in Fig. 5 show the trajectories on the phase plane, where the relative displacement $\left(x_{1}-x_{2}\right)$ is given on the horizontal axis, and the relative velocity $\left(y_{1}-y_{2}\right)$ is on the vertical axis. Numerical simulation shows that the system has a small window of period-one response with one impact (contact phase between internal mass and the plate) per period of excitation for $\gamma \in[0.1,0.185]$. As the mass ratio increases, period-one response with two impacts per period of excitation is observed for $\gamma \in(0.185,6.4]$, and then a period-one response with one impact per period of excitation for $\gamma \in(6.4,10]$ is obtained. In Fig. 5(b), the average progression of the capsule per period calculated for steady state response is presented. Our study has revealed that the maximum average progression is achieved at $\gamma=0.315$. As the mass ratio increases $(\gamma>0.315)$, the average progression decreases. This result is easy to understand, since the mass of the capsule becomes much larger than the internal mass, the motion of the mass becomes ineffective to drive the capsule.

The influence of the stiffness ratio, $\beta$ is studied next and Fig. 6(a) presents the bifurcation diagram where $\beta$ is used as a branching parameter. Again it can be observed that the system response is period-one motion for all the values of stiffness ratio. The trajectories of the mass on phase plane are shown in the additional windows in Fig. 6, where a period-one response with one impact per period of excitation for $\beta \in[0.1,8.864]$ and a period-one response with two impacts per period of excitation for $\beta \in(8.864,15]$ are presented. The average progression of the capsule per period is shown in Fig. 6(b) where the maximum average progression is observed at $\beta=8.918$. Time histories of the displacements calculated for different stiffness ratios are presented in Fig. 7. As can be seen from this figure, for a small stiffness ratio $\beta=1.5$ (Fig. $7(\mathrm{a})$ ), the secondary spring cannot generate sufficient force to enhance the progression of the capsule, and the capsule acts like vibrations absorber rather than a force transmitter. When the stiffness ratio increases, the progression of the capsule is enhanced by the impacts as shown in Fig. 7(b) and (c) for $\beta=3.5$ and $\beta=7.0$, respectively. When the mass has two impacts with the plate per period of excitation, a maximum progression is achieved as shown in Fig. 7(d) for $\beta=8.918$. Then as the stiffness increases further $(\beta>8.918)$, the interactions become less affective and the average progression per period is decreasing as observed in Fig. 6(b).

A bifurcation diagram using the frequency of excitation, $\omega$ as a branching parameter is presented in Fig. 8. It can be seen that the motion of the system is a period-one response for $\omega \in[0.1,2.225]$, and additional windows demonstrate its bifurcations from a period-one response with three impacts per period of excitation to a period-one response with one impact per period. As the excitation frequency increases, a small window of period-three response is observed for $\omega \in(2.225,2.3]$, followed by a period-two response for $\omega \in(2.3,2.575]$. And then a small window of periodfour response is recorded for $\omega \in(2.575,2.675]$ before a large region of period-two response for $\omega \in(2.675,3.675]$. For $\omega \in(3.675,3.775]$, a period-five response is observed followed by a small region of period-two response for $\omega \in(3.775,3.975]$. It is also found that there are two co-existing attractors for $\omega \in(3.909,3.928)$ where the above mentioned period-two response co-exists with period-five response which undergoes 

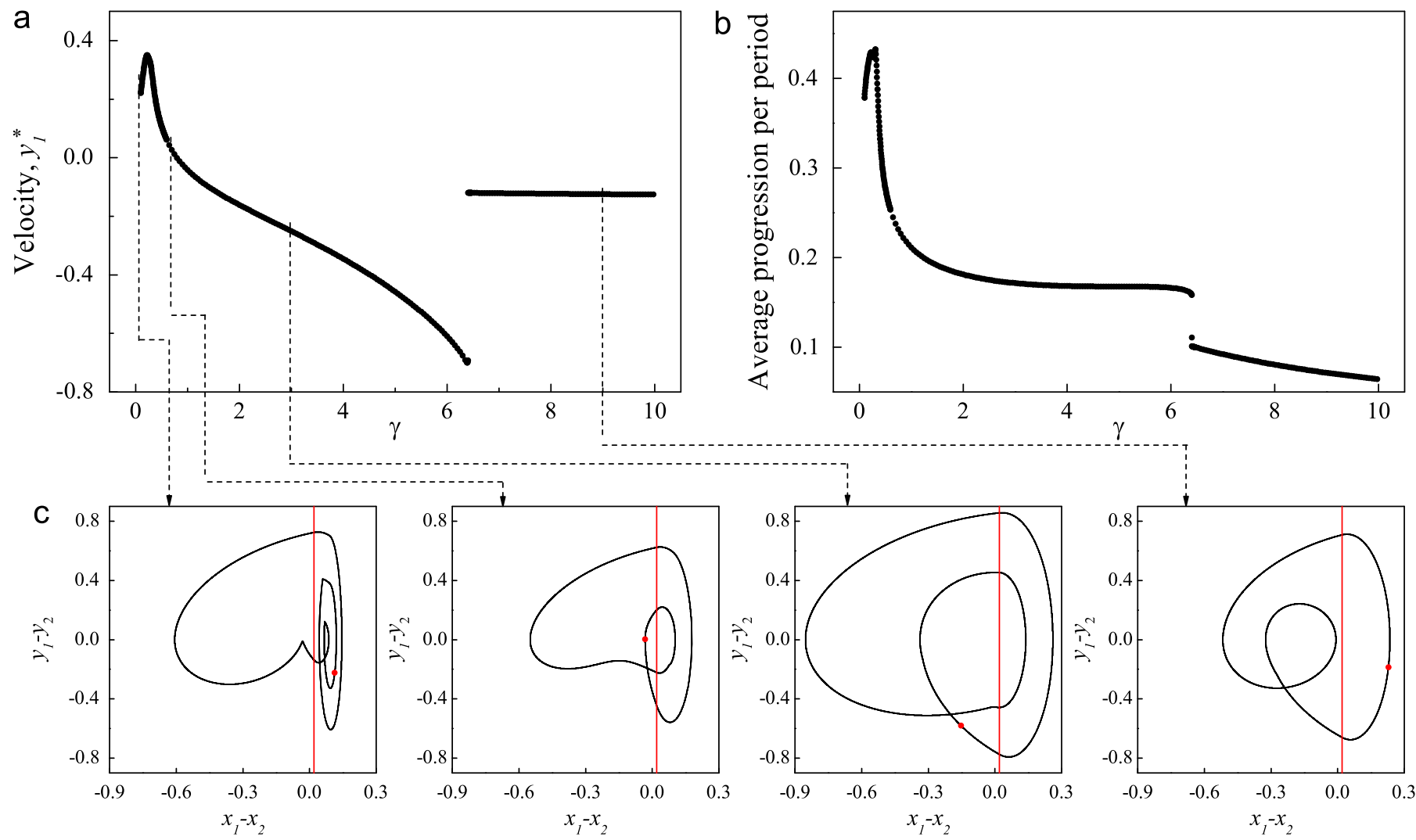

Fig. 5. (colour online) Bifurcation diagrams constructed for varying mass ratio, $\gamma$ : (a) velocity of the internal mass, $y_{1}^{*}$ and (b) average progression of the capsule per period

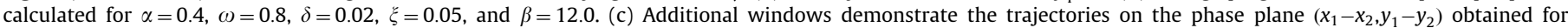
$\gamma=0.1,0.7,5.0$ and 9.0 , respectively. The locations of the impact surface are shown by red lines and Poincare sections are marked by red dots.

period-doubling bifurcations leading to chaos. Finally, a periodthree response is recorded for $\omega \in(3.975,5.0]$. From our study, we have obtained that the maximum average progression occurred at $\omega=1.675$ as shown in Fig. 9, where a period-one response with one impact per period of excitation is observed. As the excitation frequency increases $(\omega>1.675)$, the average progression decreases until the capsule becomes stationary. A small progression occurs at $\omega \in[2.25,2.3]$, where the motion of the internal mass is a periodthree response with three impacts per period of excitation. Another two local peaks of average progression are observed at $\omega=3.175$ and 4.625 , which correspond to period-two and period-three responses, respectively.

A bifurcation diagram using the amplitude of excitation, $\alpha$ as a branching parameter is shown in Fig. 10. As can be seen, a periodone response is obtained for all the values of $\alpha$, and additional windows demonstrate the transitions of its relative trajectories from a period-one response with one impact per period of excitation to a period-one response with two impacts per period as the excitation amplitude increases. A special consideration is given to the discontinuity observed for $\alpha \approx 0.2675$ where the response jumps from the period-one attractor with one impact per period to the period-one attractor with two impacts. Our numerical study reveals that these two attractors co-exist for $\alpha \in(0.2675,0.3)$ as shown in Fig. 10 (the co-existing period-one response with one impact per period is shown in red in this region) and there is a grazing bifurcation of the period-one response with two impacts per period at $\alpha \approx 0.2675$ where it ceased to exist. An interesting bifurcation is observed at $\alpha=0.618$. Although the changes in the system motion are not visible on the trajectories shown on the phase plane $\left(x_{1}-x_{2}, y_{1}-y_{2}\right)$, they are clearly seen in the time histories of capsule velocity. As can be seen in Fig. 11, for $\alpha<0.618$, the capsule has only forward motion, but for $\alpha>0.618$, the backward motion appears at every cycle. Another interesting bifurcation is observed at $\alpha=1.0$. As can be seen from typical trajectories shown in Fig. 12 , for $\alpha \in(0.2675,1.0)$ the capsule has three short stationary pauses between two progressive and one backward motions at every cycle, but for $\alpha>1.0$ two of these stationary pauses disappear.

The average progression as function of the amplitude of the excitation, $\alpha$ is presented in Fig. 13. It is clearly seen that the maximum average progression is achieved at $\alpha=1.2$, and additional windows show that the system exhibits a period-one response with two impacts per period of excitation. It is interesting to note that for this set of parameters the capsule has a large forward and a small backward motions every cycle. As the excitation amplitude increases $(\alpha>1.2)$, the average progression of the capsule decreases, and eventually the direction of the capsule motion is reversed at $\alpha \approx 1.93$ so that the negative progression of the capsule is obtained for $\alpha \in[1.93,2.0]$.

\section{Remarks on energy consumption and optimization}

In the previous section we have analysed the system dynamics and determined the parameters which provide the optimal progression per one period of external excitation. However, it is clear that the faster forward motion of the system will require higher energy input. Therefore, the successful operation of the capsule should be based on the balanced approach where both the speed of progression and the required energy are considered and taken into account. As discussed above, in some cases backward motion of the capsule could lead to decrease in average progression and it is likely to result in an increase in energy consumption. 

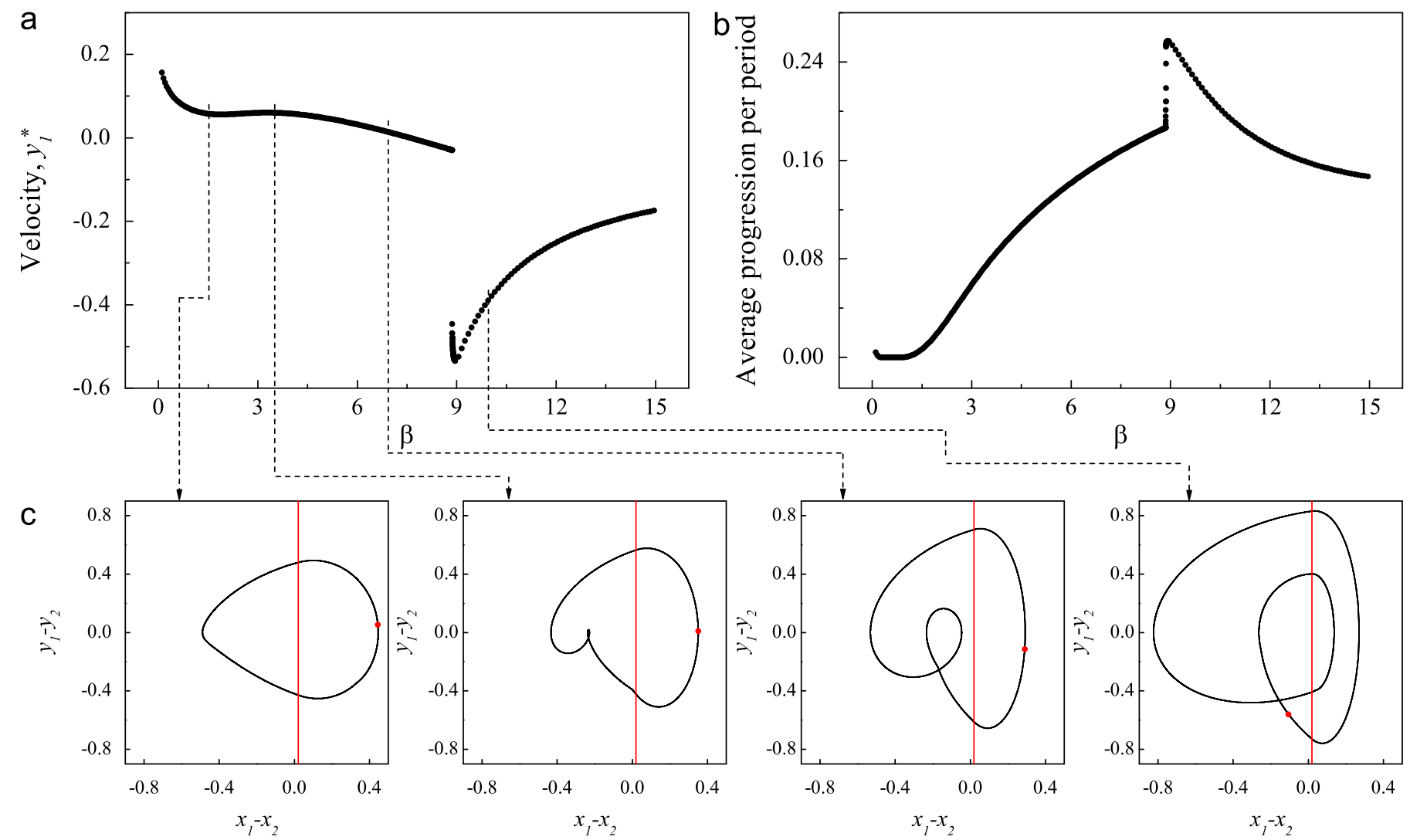

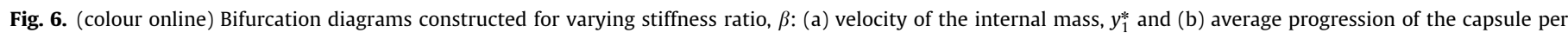

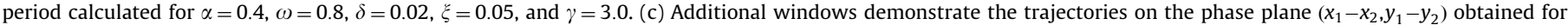
$\beta=1.5,3.5,7.0$ and 10.0, respectively. The locations of the impact surface are shown by red lines and Poincaré sections are marked by red dots.
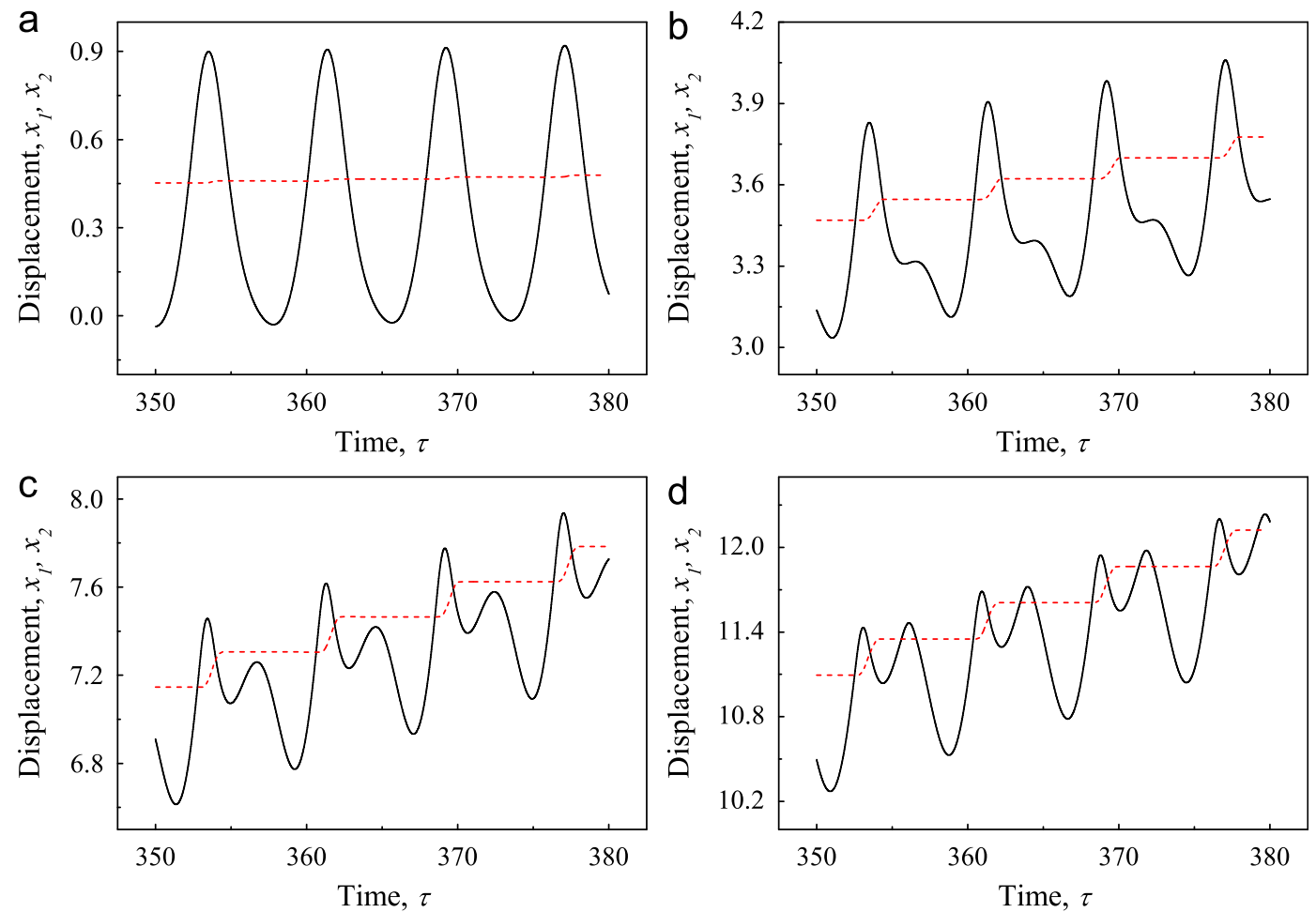

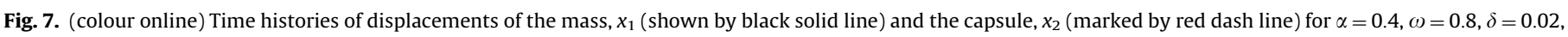
$\xi=0.05$, and $\gamma=3.0$ : (a) $\beta=1.5$; (b) $\beta=3.5$; (c) $\beta=7.0$; (d) $\beta=8.918$. 


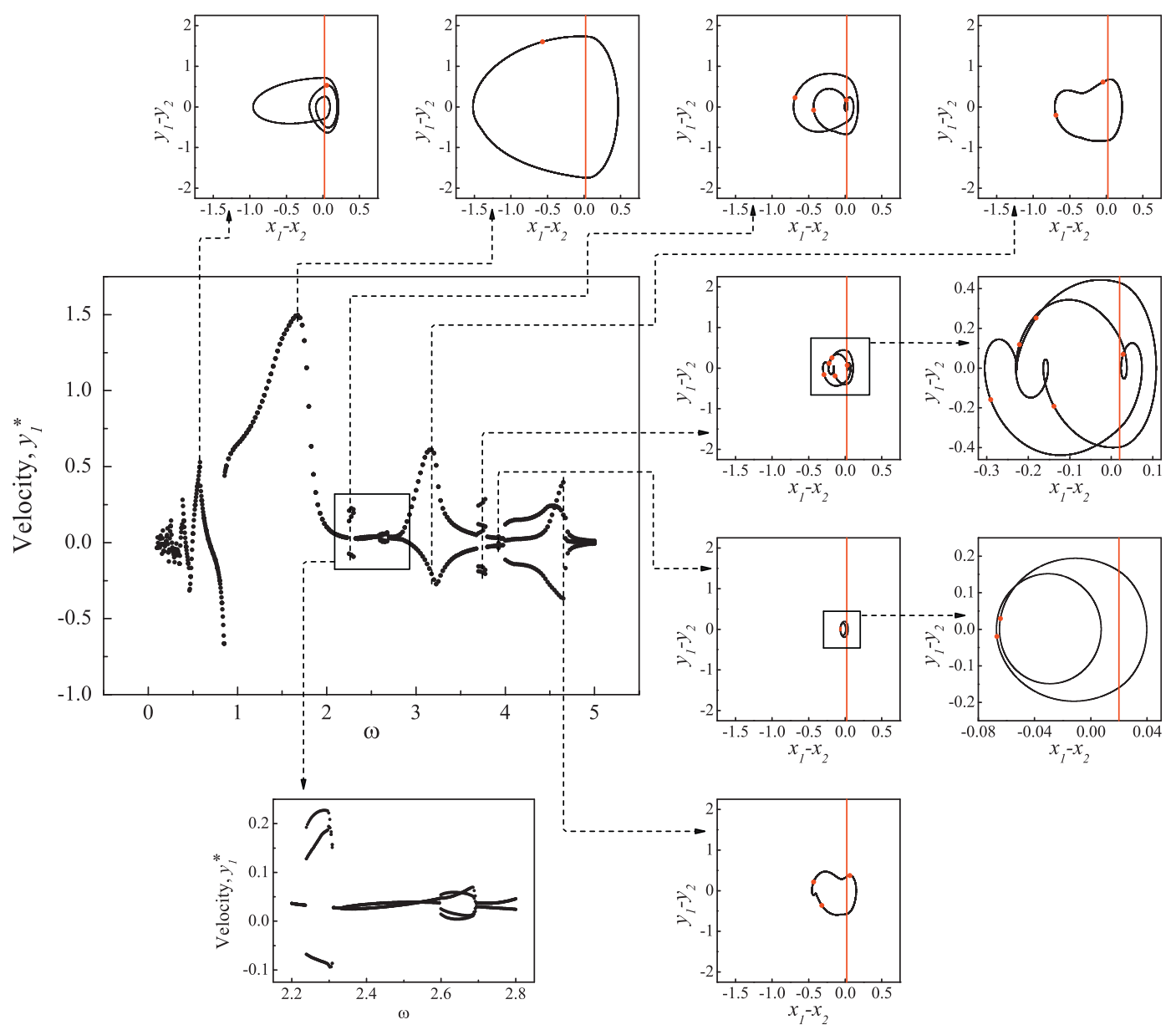

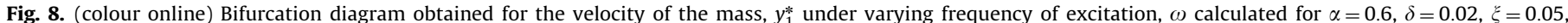

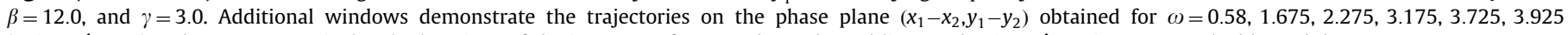
(Poincaré map) and 4.625, respectively. The locations of the impact surface are shown by red lines and Poincaré sections are marked by red dots.

In order to take the energy consumption into account, we introduce the ratio of the capsule progression per period of the external excitation, $T$ to the work done by the external force over one period

$P_{\text {avg }}=\frac{x_{2}(T)-x_{2}(0)}{\int_{0}^{T} \alpha \cos (\omega \tau) \cdot v_{1}(\tau) d \tau}$.

To optimize the system operation, we need to find out the external parameters (i.e. amplitude and frequency of the external excitation) which maximize either average progression per period or ratio $P_{\text {avg. }}$ The results of the calculations are presented in Fig. 14 (a) and (b), respectively. From Fig. 14, the maximum average progression is obtained at $\omega=1.0$ and $\alpha=2.0$, and the maximum ratio $P_{a v g}$ is achieved at $\omega=0.1$ and $\alpha=0.95$. As can be seen, the optimal control parameters from the point of view of the maximum average progression of the capsule per period are not the most efficient parameters from the energy consumption point of view, and vice versa. This difference also indicates that for designing such a capsule system, "fast" and "efficient" modes have to be considered separately for different optimization purposes.

\section{Concluding remarks}

A dynamics vibro-impact model of the capsule driving system was studied in this paper. The system consists of a capsule main body interacting with an internal mass driven by a harmonic excitation. Coulomb's friction model was used to describe the interactions of the capsule with the environment. The motion of the capsule is possible when the force acting on the capsule exceeds the threshold of the dry friction force.

Our bifurcation studies have revealed that the behaviour of the system was mainly periodic, and the best rate of progression can be achieved by a proper choice of system parameters. Investigating various mass ratio $\gamma$, it was found that for the considered set of parameters the system experienced period-one motion for all studied values of mass ratio, and the maximum average progression per period was achieved at $\gamma=0.315$. As the mass ratio increases above this value, $\gamma>0.315$, the motion of the internal mass becomes ineffective, and the average progression decreases.

Investigating various stiffness ratio $\beta$, we observed that the system response varied from period-one motion with one impact per period of loading to period-one motion with two impacts, and the maximum average progression was reached at $\beta=8.918$.

From the bifurcation study on the frequency of excitation $\omega$, we have found abundant periodic motions, and co-existing periodic and chaotic attractors. However, the capsule progression was not obtained for all frequency values. The maximum average progression was reached at $\omega=1.675$ where the capsule was in period-one motion with one impact per period of loading.

The bifurcation analysis on the amplitude of excitation $\alpha$ indicates that, a period-one response exists again for all the values of the amplitude for the considered set of parameters. As the amplitude of excitation increases, the motion of the system varies from period-one motion with one impact to period-one motion with two impacts, and these two attractors co-exist for a 

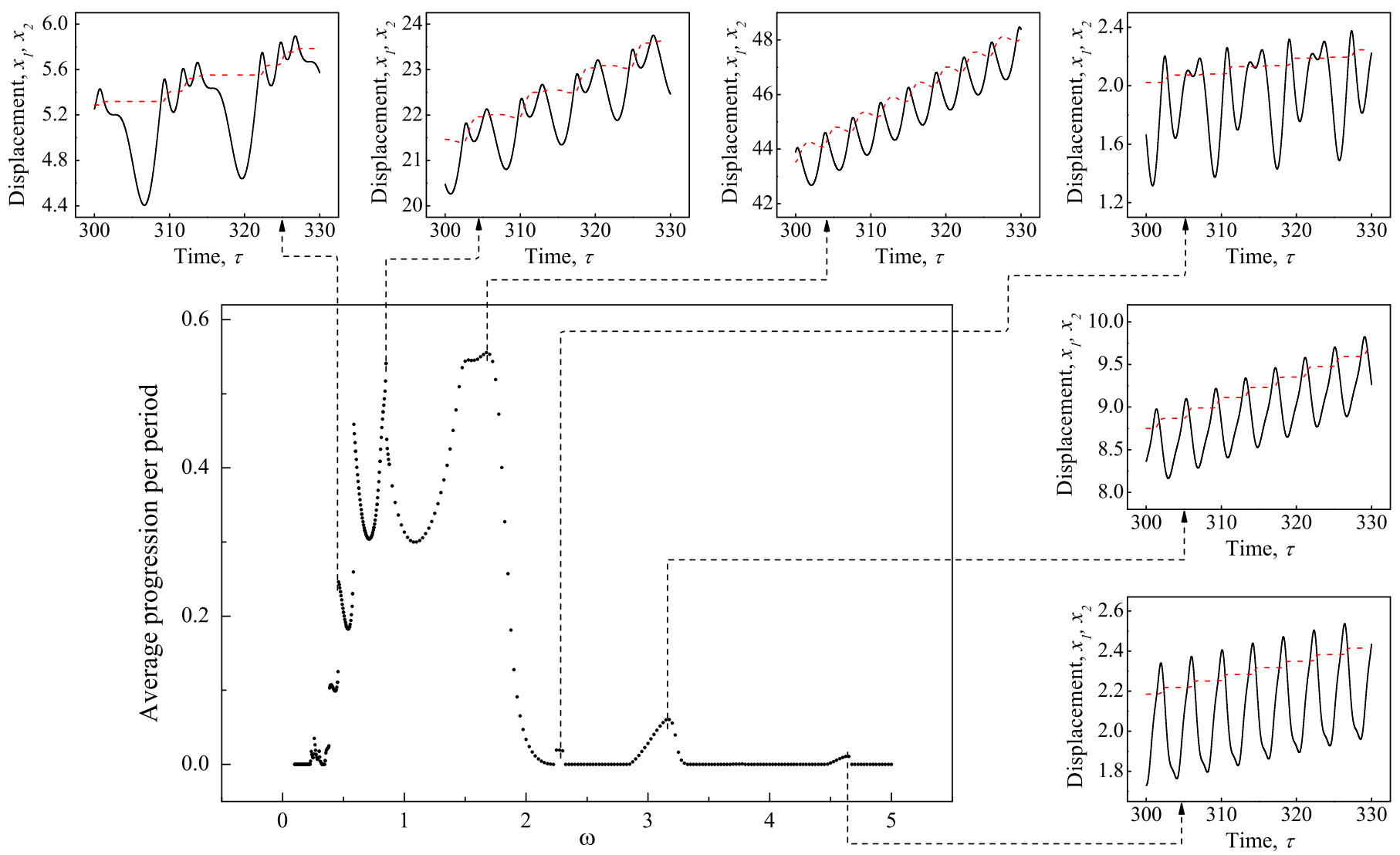

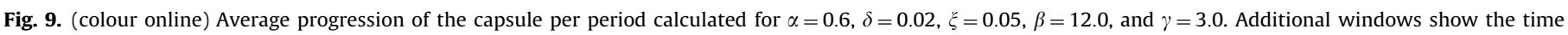

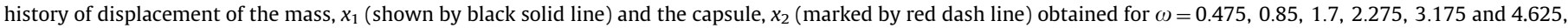
respectively.
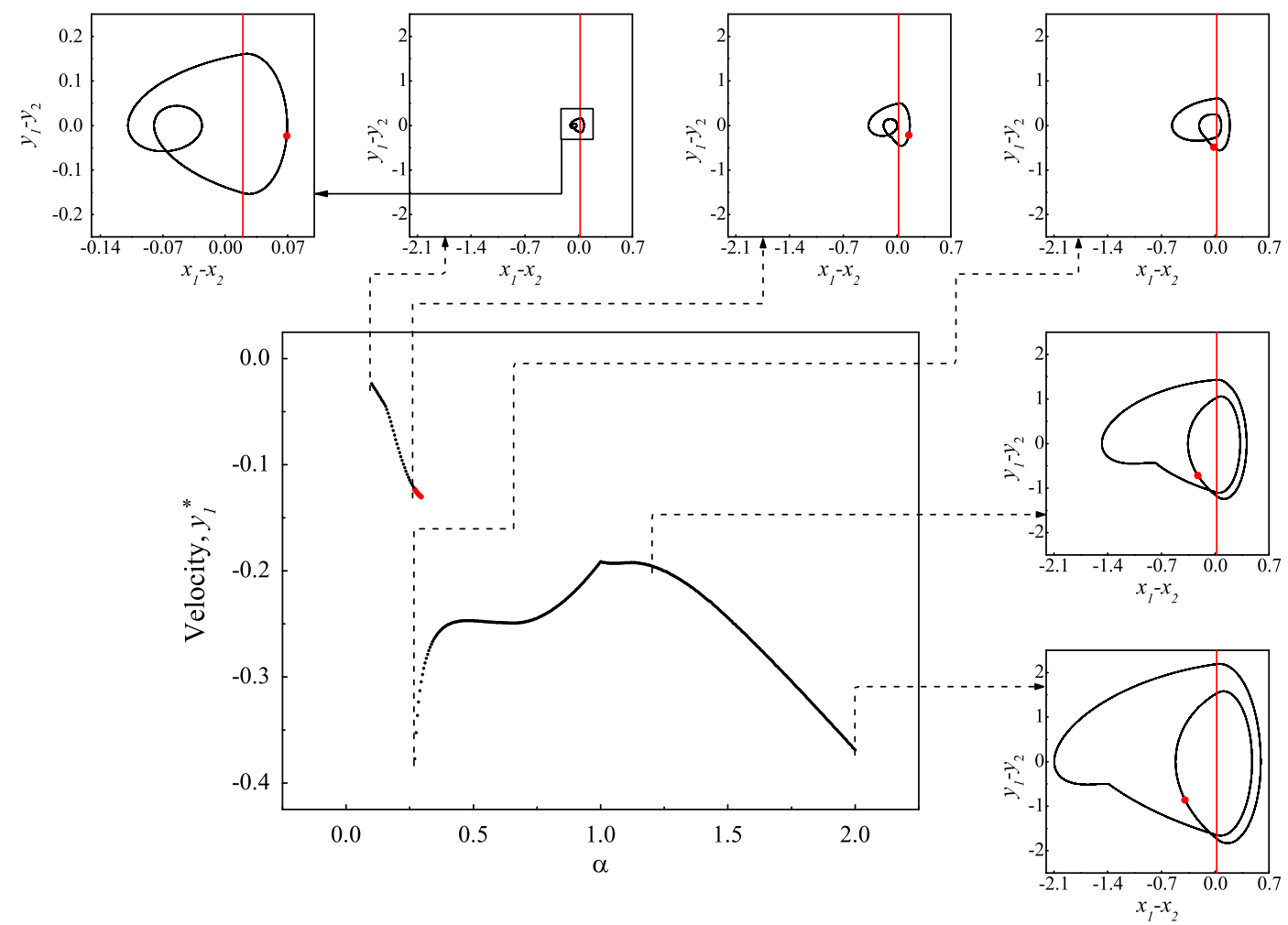

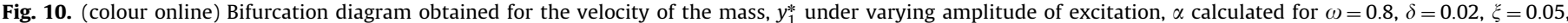

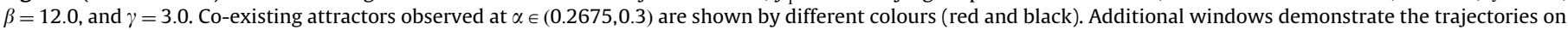

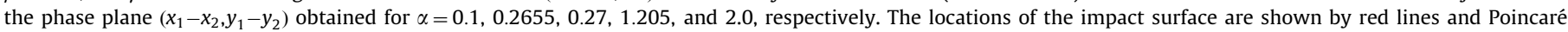
sections are marked by red dots. 

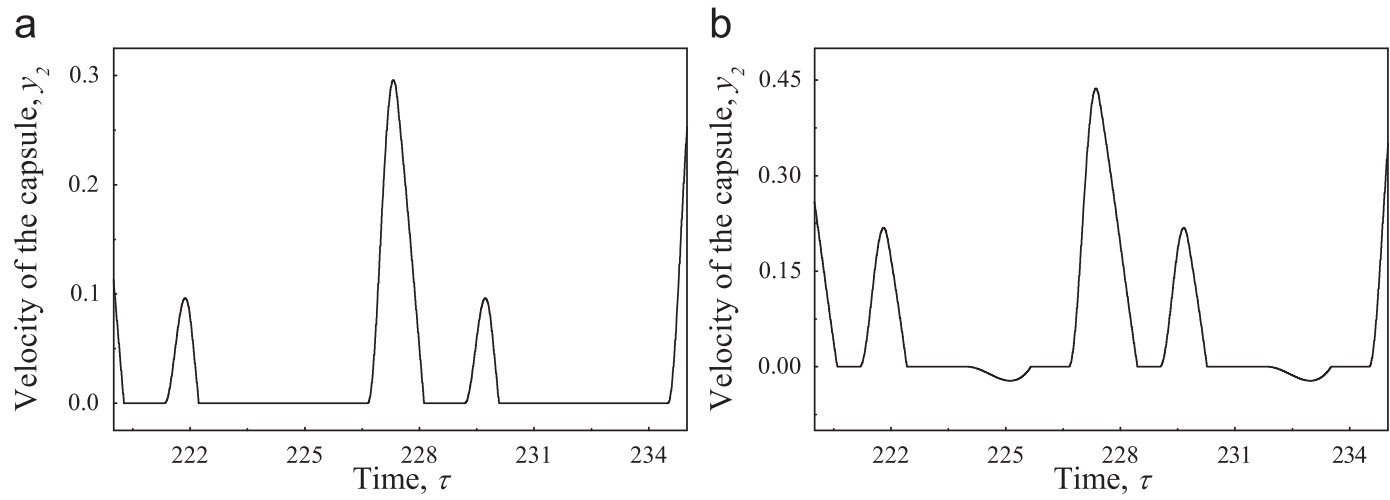

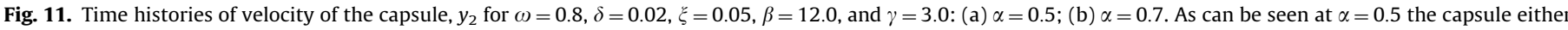
moves forward (the velocity is positive) or remains stationary whereas at $\alpha=0.7$ it can also move backwards (the velocity is negative).

a

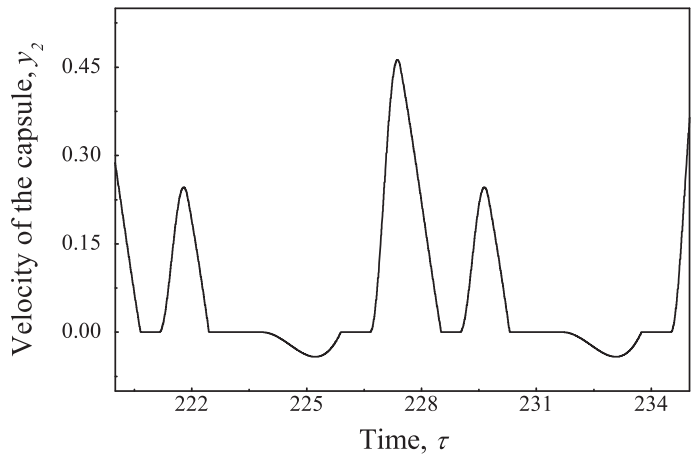

b

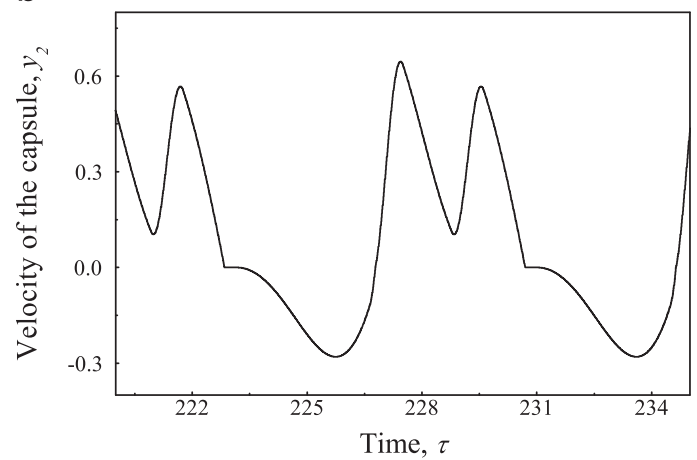

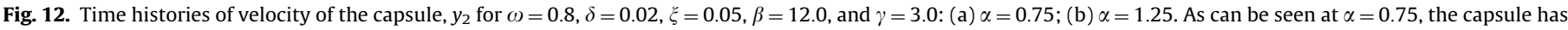
three short stationary pauses between two progressive and one backward motions at every cycle, but at $\alpha=1.25$ two of these stationary pauses disappear.

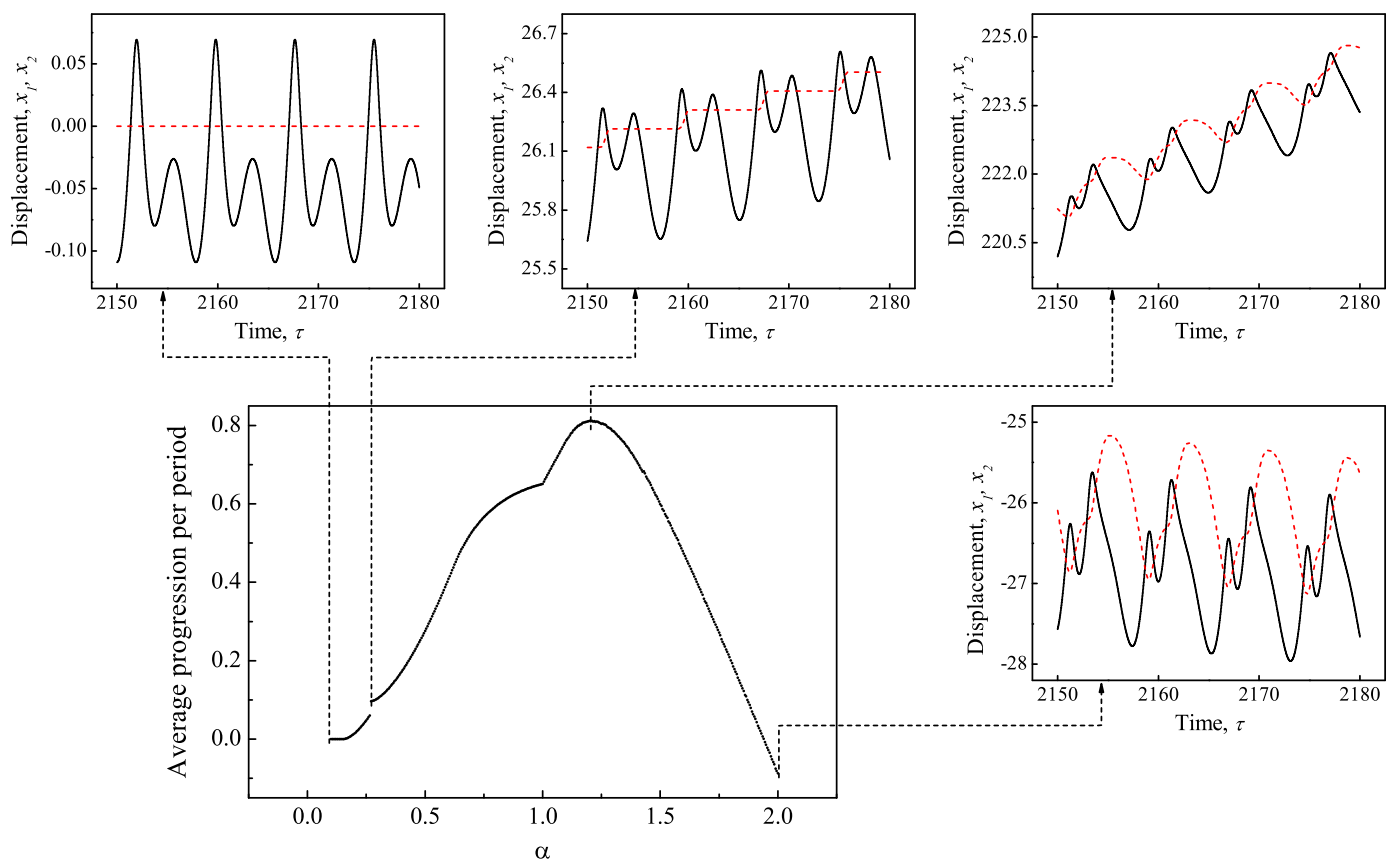

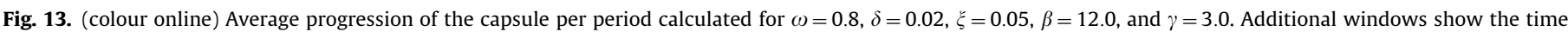

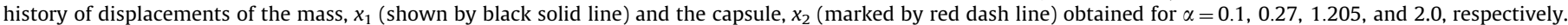

small region $\alpha \in(0.2675,0.3)$. Another two important bifurcations were observed at $\alpha=0.618$ and $\alpha=1.0$, where backward motion occurred and some intermittent pauses between capsule forward and backward motions disappeared, respectively.
From the point of view of energy consumption, we introduced the ratio of the capsule progression per period of the external excitation to the work done by the external force in one period. By calculating the ratio $P_{a v g}$, we have found that the optimum 

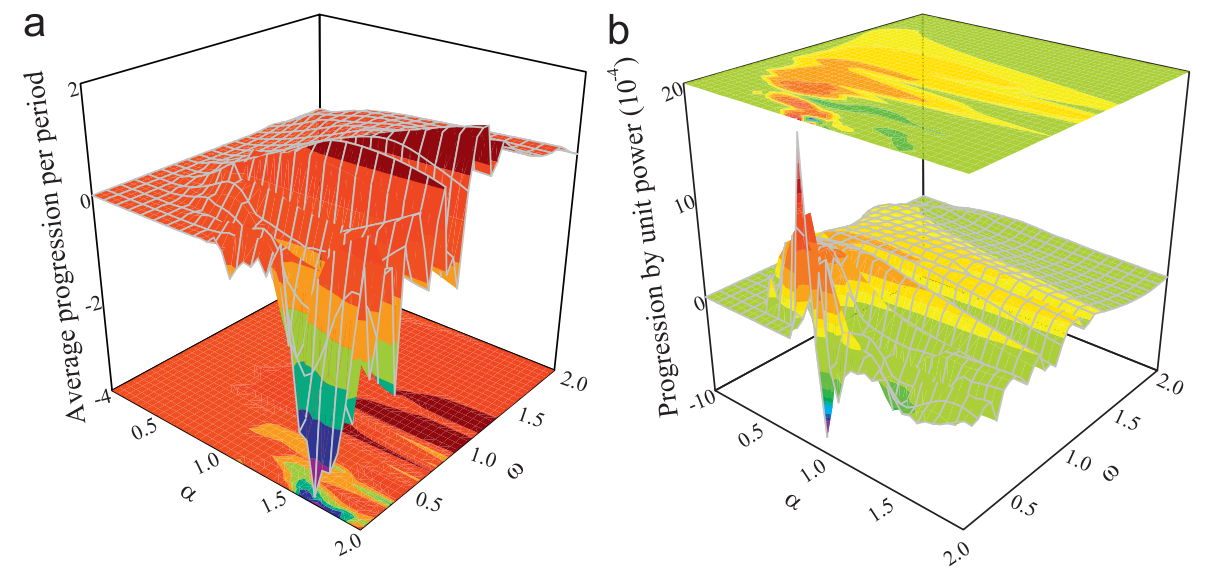

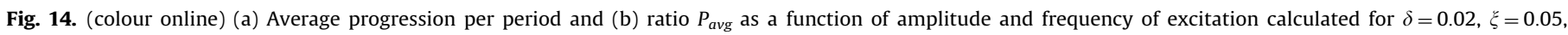
$\beta=12.0$, and $\gamma=3.0$.

parameters for the maximum progression of the capsule were not the most efficient parameters from the energy consumption point of view, and vice versa, so that a trade-off between progression and energy consumption is required for optimization.

\section{Acknowledgement}

We would like to thank Dr James Ing of the Centre for Applied Dynamics Research at the University of Aberdeen for his discussion and comments on this work.

\section{References}

[1] Chernousko FL. The optimum rectilinear motion of a two-mass system. J Appl Math Mech 2002;66:1-7.

[2] Glass P, Cheung E, Sitti M. A legged anchoring mechanism for capsule endoscopes using micropatterned adhesives. IEEE Trans Biomed Eng 2008;55:2759-67.

[3] Valdastri P, Webster R, Quaglia C, Quirini M, Menciassi A, Dario P. A new mechanism for mesoscale legged locomotion in compliant tubular environments. IEEE Trans Robotics 2009;25:1047-57.
[4] Chernousko FL. The optimum periodic motions of a two-mass system in a resistant medium. J Appl Math Mech 2008;72:116-25.

[5] Vartholomeos P. Papadopoulos E. Dynamics, design and simulation of a novel microrobotic platform employing vibration microactuators. J Dyn Syst Meas Control 2006;128:122-33.

[6] Bolotnik NN, Zeidis IM, Zimmermann K, Yatsun SF. Dynamics of controlled motion of vibration-driven systems. J Comput Syst Sci Int 2006:45:157-67.

[7] Li H, Furuta K, Chernousko FL. Motion generation of the capsubot using internal force and static friction. In: Proceedings of the 45th IEEE conference on decision and control, San Diego, CA, USA; 2006. p. 6575-80.

[8] Su G, Zhang C, Tan R, Li H. A design of the electromagnetic driver for the internal force-static friction capsubot. In: Proceedings of the IEEE/RSJ international conference on intelligent robots and systems, St. Louis, USA; 2009. p. 613-7.

[9] Liu Y, Yu H, Cang S. Modelling and motion control of a double-pendulum driven cart. Proc IMechE Part IJ Syst Control Eng. 2012;226:175-87.

[10] Fang HB, Xu J. Dynamics of a mobile system with an internal accelerationcontrolled mass in a resistive medium. J Sound Vib 2011;330:4002-18.

[11] Fang HB, Xu J. Dynamic analysis and optimization of a three-phase control mode of a mobile system with an internal mass. J Vib Control 2011;17: 19-26.

[12] Pavlovskaia E, Wiercigroch M, Grebogi C. Modeling of an impact system with a drift. Phys Rev E 2001;64:056224.

[13] Nakamura T, Terano A. Capsule endoscopy: past, present, and future Gastroenterol 2008;43:93-9.

[14] Pavlovskaia E, Wiercigroch M. Periodic solution finder for an impact oscillator with a drift. J Sound Vib 2003;267:893-911. 